EConomía Teoría y PrÁctica • Nueva Época, Número especial, vol. 4, agosto 2018, pp. 77-102, http://dx.doi.org/10.24275/ETYPUAM/NE/E042018/Salazar

\title{
Impacto del consumo de energía y del valor agregado de las manufacturas en el crecimiento económico de los países que integran el TLCAN: un modelo de datos panel cointegrado con cambio estructural*
}

\section{Impact of Electric Power Consumption and Manufacturing Added Value on Economic Growth of the Member Countries of NAFTA: a Cointegrated Panel Data Model with Structural Change}

\author{
Héctor F. Salazar-Núñez** y Francisco Venegas-Martínez***
}

\section{RESUMEN}

Esta investigación estudia las relaciones entre el consumo de energía, el valor agregado de las manufacturas y el crecimiento económico para los países integrantes del Tratado de Libre Comercio de América del Norte (TLCAN), antes y después de la firma de este tratado. Para ello se utiliza la metodología de datos panel cointegrados con cambio estructural durante el periodo 1970-2015. El método de estimación utilizado es el de mínimos cuadrados ordinarios totalmente modificados. La evidencia empírica encontrada sugiere que el valor agregado de las manufacturas mantiene una relación causal de tipo bidireccional con el producto interno bruto (PIB) en el corto plazo y neutral con el consumo de energía. Sin embargo, en el largo plazo las variables explicativas tienen una relación causal unidireccional hacia el PIB. Por último, se analiza la estabilidad estructural del modelo en el largo plazo, mediante la estimación de los residuos recursivos, encontrando que después de la firma del TLCAN se presentó un cambio estructural.

Palabras clave: Crecimiento económico, energía eléctrica, innovación tecnológica, modelos de datos panel. Clasificación JEL: F43, O13, Q55, C33

\section{Abstract}

This paper is aimed at studying the relationships among the energy consumption, the value added of manufactures, and economic growth for the countries integrating NAFTA before and after the North American Free Trade Agreement (NAFTA). To do this, we use a panel data-based cointegration approach with structural change during 1970-2015. The estimation method used is the fully modified ordinary least squares. The found empirical evidence suggests that the value added of manufactures maintains a bi-directional causal relationship with GDP and neutral with energy consumption in the short run. However, in the long run explanatory variables have a unidirectional causal relationship towards GDP. Finally, the structural stability of the model is analyzed in the long term, by means of the estimation of the recursive residues, finding that after the signing of NAFTA a structural change was presented.

Keywords: Economic growth, electrical energy, technological innovation, panel data models, manufacture industry.

JEL Classification: F43, O13, Q55, C33

*Fecha de recepción: 30/06/2017. Fecha de aprobación: 12/12/2017.

** Universidad Panamericana. Correo: hectorsalazar313@gmail.com. ORCID: 0000-0002-2495-7646

***Instituto Politécnico Nacional. Correo: fvenegas1111@yahoo.com.mx. ORCID: 0000-0002$1528-5593$ 


\section{INTRODUCCIÓN}

El impacto del uso de la energía y la innovación tecnológica en el crecimiento económico del Producto Interno Bruto (PIB) en los países que integran el Tratado de Libre Comercio de América del Norte (TLCAN) es un tema relevante de actualidad, ya que la energía y la innovación (incluida en la cadena de valor agregado de la industria manufacturera) contribuyen a la expansión de bienes y servicios, incrementando con ello la productividad del sector manufacturero mediante el uso de maquinaria, equipo y tecnología.

La relación existente entre el uso de energía y el PIB en el largo plazo ha sido un tema de continuo debate desde que se publicó el artículo seminal de Kraft y Kraft (1978), quienes buscaban relaciones de largo plazo entre el uso de la energía y el PIB en los Estados Unidos. ${ }^{1}$ Por otro lado, la importancia de la actividad innovadora en el crecimiento económico se enfatiza desde hace mucho tiempo en los trabajos de Schumpeter (1911), Kuznets (1966), Parsons (1968) y Schmookler (1966), entre muchos otros.

La presente investigación examina la existencia de relaciones de largo plazo entre el valor agregado de las manufacturas y el crecimiento económico para los países integrantes del TLCAN. De acuerdo con Aghion et al. (2004), la productividad total de los factores también se explica por otros factores distintos al trabajo y al capital (el residual de Solow). Entre estos factores se incluyen el avance tecnológico, la inversión en I+D (investigación y desarrollo), las patentes y la exportación de productos de alto contenido tecnológico. De esta forma, el valor agregado en la industria de las manufacturas, además de salarios, posee un contenido de innovación tecnológica. México tiene una manufactura avanzada de gran valor agregado que impacta su crecimiento. Canadá y Estados Unidos en mayor medida obtienen grandes beneficios de esto por medio de una gran cadena de abastecimiento y de un régimen de políticas favorables, con salarios competitivos, para la industria manufacturera; siendo los clústeres productivos en las ramas automotriz y de aparatos y componentes electrónicos, un objetivo primario. ${ }^{2}$

En esta investigación, las posibles relaciones entre el uso de energía eléctrica, el valor agregado de las manufacturas y el crecimiento del producto se estudian con

${ }^{1}$ Kraft y Kraft (1978) utilizan la prueba de causalidad de Sims (1972). Desde entonces, muchas investigaciones han buscado relaciones entre el crecimiento del PIB y el uso de energía con diferentes metodologías; entre las más populares se tienen las desarrolladas por Engle y Granger (1987) y Johansen (1988) y (1991). Otros trabajos pioneros relacionados con energía y PIB se pueden encontrar en Tatom (1981) y Denison (1985).

${ }^{2}$ General Motors de México, Chrysler de México y Ford de México. 
base en un modelo de datos panel agrupados (pooled) utilizando la metodología de cointegración. Esta metodología aplicada a modelos de datos panel requiere de: 1) pruebas de estacionariedad (o de raíz unitaria), 2) pruebas de cointegración y 3) métodos de estimación de parámetros. Por el lado de las pruebas de estacionariedad para series de tiempo se utilizan las desarrolladas por Dickey y Fuller (1979), Phillips y Perron (1988), Kwiatkowski, Phillips, Schmidt y Shin (1992), mientras que para datos panel se utilizan las propuestas de Maddala y Wu (1999), Hadri (2000), Choi (2001), Levin, Lin y Chu (2002) e Im, Pesaran y Shin (2003), aunque existen muchas otras. Entre las pruebas de cointegración para datos panel destacan las propuestas de Pedroni (1996, 1999, 2001 y 2004) basadas en Engle y Granger (1987), Maddala y Wu (1999), Fisher (1932), Johansen (1988, 1991), Phillips y Hansen (1990), Phillips y Moon (1999) y Kao y Chiang (2000). En conjunto estos tres pasos conforman la metodología de cointegración de datos panel o datos panel cointegrados. La aplicación de esta metodología puede conducir a tres casos: primero, que exista una relación bidireccional, es decir, la relación causal es en ambas direcciones; segundo, que sólo exista una relación unidireccional, y, tercero, que no exista relación alguna, es decir, se tiene una relación neutral.

Con respecto a la búsqueda de relaciones entre energía, innovación y PIB, Tang y Tan (2013) estudian la relación causal entre el consumo de electricidad, los precios de la energía, la innovación tecnológica y el crecimiento económico en Malasia durante el periodo 1970-2009, encontrando que la innovación tecnológica tiene una relación causal con el crecimiento económico y el consumo de la electricidad. Por otro lado, estos autores encontraron una relación bidireccional entre el consumo eléctrico y el crecimiento económico. Asimismo, Coers y Sanders (2013) analizan el uso de la energía en términos per cápita, la formación bruta de capital (FBK), la cual tiene un contenido parcial de innovación tecnológica, y el PIB de los países pertenecientes a la Organización para la Cooperación y el Desarrollo Económicos (OCDE) mediante un modelo panel de datos y el uso de la metodología de cointegración en el periodo 1960-2000, encontrando que en el corto plazo las variables de estudio mantienen una relación causal de tipo bidireccional y en el largo plazo la FBK y el PIB explican los movimientos del uso de la energía. Asimismo, Bhattacharya, Paramati, Ozturk y Bhattacharya (2016) estudian a los 38 países de mayor consumo de energía renovable durante el periodo 1991-2012, encontrando relación causal de corto y largo plazo entre el PIB real, las tecnologías de la producción y el consumo de energía (renovable y no renovable) por medio de un modelo de datos panel cointegrado. Asimismo, estos autores encuentran que en el corto plazo las tecnologías de la producción tienen una relación unidireccional con el crecimiento económico. Por último, Destek (2016) estudia la relación causal entre la apertura comercial, el consumo de gas natural, la FBK y el crecimiento económico de 26 países pertenecientes 
a la OCDE, durante 1991-2013, con base en un modelo de cointegración y utilizando el método de estimación mínimos cuadrados ordinarios totalmente modificados (мСОтм). Los autores encuentran una relación unidireccional entre el consumo de gas natural y el crecimiento económico, mientras que las otras variables tienen una relación bidireccional al 10 por ciento de significancia en el corto plazo. En el largo plazo todas las variables mantienen una relación bidireccional al 10 por ciento.

Existen diversos trabajos que buscan relaciones de largo plazo entre el crecimiento económico y el consumo de la energía en sus diferentes formas, la FBK, las exportaciones, etcétera. Por ejemplo, Alí Aali-Bujari, Venegas-Martínez y Palafox-Roca (2017) examinan mediante un modelo dinámico de datos panel la relación entre el uso de energía y el crecimiento en las economías más grandes de la OCDE. De igual forma, Fatai, Les Oxley y Scrimgeour (2004) estudian relaciones de largo plazo entre el crecimiento económico y el consumo de la energía, la FBK y las exportaciones. Asimismo, Yoo (2005) estudia la relación causal entre el consumo eléctrico y el PIB de la economía de Corea del Sur, para el periodo 19702002, por medio de un modelo de corrección de errores, encontrando una relación bidireccional entre las variables. Salazar-Núñez y Venegas-Martínez (2018) analizan el impacto del uso de energía y la FBK en el crecimiento económico mediante un modelo de datos panel en 73 países agrupados por nivel de ingreso. Por su parte, Narayan y Prasad (2008) estudian a los países que pertenecen a la OCDE, durante el periodo 1971-2002, buscando una relación causal entre el consumo de electricidad industrial y el PIB real en 22 de las economías de la OCDE. Yuan, Kang, Zhao y Hu (2008) analizan la relación causal entre el consumo de electricidad, petróleo y el PIB de China, utilizando la metodología de cointegración de Johansen por medio de un modelo de vectores de corrección de errores para el periodo 1963-2005, encontrando una relación unidireccional de las variables explicativas y el PIB. Akinlo (2009) analiza la relación entre el consumo de energía eléctrica y el crecimiento económico de Nigeria para el periodo 1980-2006, encontrando una relación unidireccional del consumo de energía eléctrica al PIB real. Ozturk (2010) realiza una revisión de la literatura de los trabajos que estudian relaciones causales del consumo de energía y el PIB durante 1978-2009. Por su parte, Shahbaz, Tang y Shabbir (2011) examinan la relación causal entre el consumo de electricidad, el empleo y el crecimiento económico en Portugal utilizando un modelo de panel de datos con corrección de errores para el periodo 19712009, y encuentran que en el corto plazo el empleo y el crecimiento económico tienen una relación bidireccional y en el largo plazo el crecimiento económico explica los movimientos del consumo de electricidad.

Por otro lado, Apergis y Payne (2010) estudian a países emergentes consumidores de carbón, en el periodo 1980-2006, usando un modelo de datos panel y en- 
contrando una relación bidireccional entre el uso del carbón y el PIB. Ghosh (2010) estudia a 24 países pertenecientes a la OCDE, durante 1960-1997, con el fin de encontrar relaciones entre el PIB real, las exportaciones en términos reales y el nivel de apertura de la economía, habiendo obtenido resultados mixtos al aplicar la prueba de causalidad de Granger. Ozcan (2013) analiza la relación entre la emisión de monóxido de carbono, el consumo de energía y el crecimiento económico de 12 países del Medio Oriente para el periodo 1990-2008, dando con una relación unidireccional entre el consumo de energía y el crecimiento económico hacia las emisiones de monóxido de carbono. Kasman y Duman (2015) examinan la relación entre el consumo de energía, las emisiones de monóxido de carbono, el crecimiento económico y el comercio abierto de países y candidatos de la Unión Europea, en el periodo 1992-2010, encontrando relaciones de corto plazo y largo plazo de tipo unidireccional de todas las variables hacia el comercio abierto. Por último, Rezitis y Ahammad (2016) realizan un resumen de trabajos que relacionan el consumo de la energía y el PIB de países asiáticos hasta 2015 y otras regiones geográficas hasta 2013. Estos autores analizan algunas economías asiáticas, en 1990-2012, utilizando un modelo de datos panel con corrección de errores encontrando que el crecimiento económico de estas economías depende del uso de la energía.

Esta investigación analiza la relación que existe entre el crecimiento económico, el consumo de la energía eléctrica y el valor agregado de las manufacturas de los países miembros del TLCAN. La segunda sección presenta la metodología econométrica, la cual consta de pruebas de raíz unitaria y de cointegración, y el método de мсотм. En la tercera sección se realiza la aplicación del modelo descrito en la sección anterior, y se discuten los resultados empíricos obtenidos. Por último, en la cuarta sección, se presentan las conclusiones y se mencionan las limitaciones de la metodología utilizada.

\section{Metodología ECONOMÉTRICA}

Para examinar los efectos del consumo de energía y del valor agregado de las manufacturas en el crecimiento económico de los países que integran el TLCAN se utilizarán datos anuales del PIB per cápita, el consumo de energía eléctrica (CEE) en KWH per cápita y el valor agregado de las manufacturas per cápita (VAM), en el periodo 1970-2015, en dólares estadounidenses con base en 2010. De aquí en adelante se hablará del PIB para referirse al PIB per cápita a menos que se mencione lo contrario. Los datos fueron obtenidos de la Organización para la Cooperación y el Desarrollo Económicos (OCDE) y el Banco Mundial (BM).

En esta investigación las variables independientes serán CEE y VAM de los países que integran el TLCAN y se propone la siguiente relación funcional: 


$$
P I B=f(C E E, V A M)
$$

La variable VAM se introduce para representar la innovación, dada la definición de valor agregado y de manufacturas. Por supuesto, existen otras muchas formas de representar la innovación; por ejemplo, índices que genera la Organización Mundial de la propiedad Intelectual (OMPI), la Universidad de Cornell y el Instituto Europeo de Administración de Empresas (INSEAD, por sus siglas en francés), que consideran para el cálculo de sus índices el número de patentes solicitadas, el número de publicaciones científicas, las tecnologías de información, la inversión en investigación y desarrollo, así como el gasto en educación, etcétera. Otros índices los publican el Grupo de Consultoría Boston (BCG), la Asociación Nacional de Manufactureros (NAM) y el Instituto de Manufacturas (MI), los cuales se basan en el enfoque de insumo-producto. Del lado del producto se considera la educación, mientras que del lado del insumo, las patentes y la investigación y el desarrollo. También es importante mencionar el índice que publica Bloomberg y que considera: investigación y desarrollo, manufacturas y empresas de alta tecnología. No obstante todas estas posibilidades, este trabajo toma en cuenta que en México se ha desarrollado una manufactura avanzada con un gran valor agregado para las economías del TLCAN. ${ }^{3}$ De esta manera, la especificación del modelo de datos panel está dada por:

$$
P I B_{i t}=\alpha_{1}+\alpha_{2} C E E_{i t}+\alpha_{3} V A M_{i t}+\varepsilon_{i t}
$$

donde $\alpha_{1}$ es el parámetro que define la componente autónoma del modelo, $\alpha_{2}$ y $\alpha_{3}$ son los parámetros que le dan forma a las pendiente sobre cada plano, $\varepsilon_{i t}$ es una perturbación estocástica que se distribuye normalmente con media cero y varianza constante $\left(\varepsilon_{i t} N\left(0, \sigma_{\varepsilon}^{2}\right)\right)$. El subíndice $i$ representa las secciones cruzadas (en este caso MEX, CAN y USA) y $t$ representa el tiempo con una frecuencia anual durante 1970-2010.

\section{I.1. Pruebas de raíces unitarias}

La metodología que se utilizará en este estudio es la de cointegración de Engle-Granger (1987), la cual se extiende para datos panel. Para ello, primero, se realizarán

\footnotetext{
${ }^{3}$ En el caso de México la industria manufacturera aporta casi el 19 por ciento del PIB con un impulso notable hacia la innovación.
} 
pruebas de raíz unitaria o de estacionariedad de: Levin, Lin y Chu (2002), Hadri (2000), Im, Pesaran y Shin (2003), Maddala y Wu (1999), y Choi (2001). Estas pruebas se basan en las de Dickey y Fuller aumentada (1979), Phillips y Perron (1988), y Kwiatkowski, Phillips, Schmidt y Shin (1992). De aquí en adelante se hará referencia a ellas como ADF, PP y KPSS, respectivamente.

En particular, Levin, Lin y Chu (2002) proponen una prueba de raíz unitaria para datos panel, la cual se puede dividir en tres etapas. En la primera se estiman los modelos de cada sección cruzada con la prueba ADF para calcular la desviación estándar del modelo de largo plazo. En la segunda etapa se obtienen los residuos, los cuales son base para el modelo agrupado (pooled). En la última etapa se estima el modelo agrupado. Conjuntando estas tres etapas se realiza la prueba que tiene como hipótesis nula y alternativa $H_{0}: \alpha_{i}=1$ y $H_{1}: \alpha_{i}<1$, respectivamente. De esta forma, todas las secciones cruzadas tienen raíces comunes. El estadístico de prueba está dado por:

$$
t_{\alpha}=\frac{t_{\alpha}-\frac{N \tilde{T} S_{i} \sigma_{\hat{\alpha}} \mu_{m \tilde{T}}}{\hat{\sigma}_{\varepsilon}^{2}}}{\sigma_{m \tilde{T}}} N(0,1)
$$

donde $t_{\alpha}$ es el estadístico $t$ de Student del modelo agrupado, $N \tilde{T}$ son el número total de observaciones, $S_{i}=(1 / N)\left(\sum_{i} s_{i}\right)$ es la razón promedio de las desviaciones estándar (es la razón de la desviación estándar y la covarianza consistente con heterocedasticidad y autocorrelación de las regresiones individuales de la prueba ADF), $\hat{\sigma}_{\varepsilon}^{2}$ es la varianza estimada de $\varepsilon$ del modelo agrupado, $\sigma_{\hat{\alpha}}$ es el error estándar de $\hat{\alpha}$ del modelo agrupado, $\mu_{m \tilde{T}}$ y $\sigma_{m \tilde{T}}$, son la media y la desviación estándar ajustadas que dependen de la serie de tiempo de tamaño $\tilde{T}$ del modelo agrupado, $\tilde{T}=T-\left(\sum_{i} p_{i} / N\right)-1$, con $p_{i}$ igual al número de rezagos de cada modelo.

Asimismo, Hadri (2000) propone una prueba de estacionaridad para el modelo datos panel que se basa en la prueba propuesta por Kwiatkowski, Phillips, Schmidt y Shin (1992), donde la ecuación estimada puede estar en función de una constante o tendencia determinista, o ambas. Para probar la hipótesis nula de estacionariedad se utiliza: 


$$
L M=\frac{1}{N}\left(\frac{\sum_{i=1}^{N}\left(\frac{\sum_{t=1}^{T} S_{i t}^{2}}{T^{2}}\right)}{\hat{\sigma}_{\varepsilon, i}^{2}}\right)
$$

donde $S_{i t}^{2}=\sum_{j=1}^{t} \hat{\varepsilon}_{i j}$, es la suma parcial de los residuos, $\hat{\sigma}_{\varepsilon, i}^{2}=\sum_{i=1}^{N} \sum_{t=1}^{T} \hat{\varepsilon}_{i t}^{2} / N(T-k)$ es el promedio de los estimadores individuales del espectro residual de frecuencia cero (con covarianza consistente con heterocedasticidad y autocorrelación) similar a la varianza de largo plazo (una segunda forma de calcular $\hat{\sigma}_{\varepsilon}^{2}$ se tiene cuando $i=0$ ), la cual es consistente con el número $k$ de variables exógenas. En este caso se utiliza la propiedad:

$Z=\frac{\sqrt{N}(L M-\xi)}{\zeta} \rightarrow N(0,1)$

donde $\xi=E\left[\int_{0}^{1} V(r)^{2} d r\right], \zeta=\operatorname{var}\left[\int_{0}^{1} V(r)^{2} d r\right]$ y $N$ es el número de secciones
cruzadas.

De igual forma, Im, Pesaran y Shin (2003) proponen una prueba basada en la prueba ADF. En este caso, no todas las raíces son iguales bajo la hipótesis alternativa, es decir, bajo la hipótesis en cuestión se supone que una parte de las series individuales son estacionarias y otras no $\left(H_{0}: \alpha_{i}=0\right.$, para toda $i ; H_{1}: \alpha_{i}=0$ para toda $i=1,2, \ldots, N_{1} ; H_{1}: \alpha_{i}<0$ para toda $\left.i=N+1, N+2, \ldots N\right)$. En este caso se utiliza:

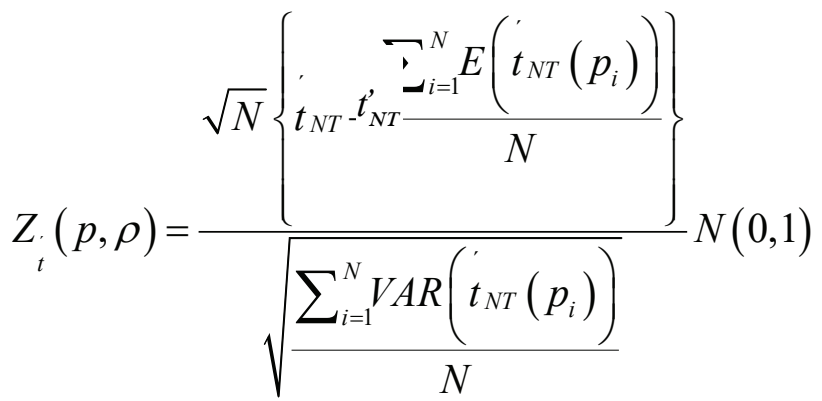


donde $\mathrm{t}_{\mathrm{NT}}$ representa el promedio del conjunto de pruebas individuales ADF, $E(\cdot)$ y $\operatorname{VAR}(\cdot)$ representan la media y la varianza promedio del estadístico ADF basado en las observaciones $T$. Observe que la función de distribución para obtener los valores $E(\cdot)$ y $\operatorname{VAR}(\cdot)$ cambia con el tiempo. Si no se utiliza la tendencia en la regresión, ésta converge a los valores de la prueba ADF, es decir, si $T \rightarrow \infty$, entonces para cada $i$ el estadístico $t$ converge a $\eta_{i}$, el cual está dado por:

$$
\eta_{i}=\frac{\frac{1}{2}\left\{\left[W_{i}(1)\right]^{2}-1\right\}-W_{i}(1) \int_{0}^{1} W_{i}(u) d u}{\int_{0}^{1}\left[W_{i}(u)\right]^{2} d u-\left[\int_{0}^{1} W_{i}(u) d u\right]^{2}}
$$

donde $W_{i}$ es el movimiento browniano estándar. ${ }^{4}$

Maddala y Wu (1999), y también Choi (2001), proponen de forma independiente una prueba de raíz unitaria para el modelo datos panel, tomando como base las pruebas ADF y PP, y las probabilidades de éstas como resultado de la suma de los diferentes niveles de significancia, ${ }^{5}$ donde las hipótesis son $H_{0}: \alpha_{i}=1 \mathrm{y}$ $H_{1}: \alpha_{i}<1$ (la hipótesis alternativa propone que no todas las secciones cruzadas tienen raíz unitaria):

ADF-Fisher $\chi^{2}$ y PP-Fisher $\chi^{2}=-2 \sum_{i=1}^{N} \log \left(\pi_{i}\right) \rightarrow \chi_{2 N}^{2}$

donde $\pi_{i}$ es el $p$-value para cada prueba individual de ADF o PP para la sección cruzada $i$. En este caso:

ADF-Choi y PP-Choi $Z=\frac{\sum_{i=1}^{N} \Phi^{-1}\left(\pi_{i}\right)}{N} \rightarrow N(0,1)$

donde $\pi_{i}$ es el $p$-value para cada prueba individual de ADF o PP para la sección cruzada $i$ y $\Phi^{-1}$ es la inversa de la función de distribución de una variable aleatoria normal estándar.

${ }^{4}$ Cuando se incluye la tendencia, puede consultarse a Hamilton (1994) para un desarrollo más profundo.

${ }^{5}$ Método propuesto por Fisher (1932). 


\section{I.2. Pruebas de cointegración}

Pedroni (1999 y 2004) propone una prueba de cointegración para datos panel, basándose en la metodología de Engle y Granger (1987), para lo cual deriva siete estadísticas que analizan el comportamiento de los residuos de la regresión. Las primeras cuatro estadísticas se conocen simplemente como estadísticos del panel de cointegración (dentro de dimensión) y las restantes tres como estadísticos del panel de cointegración del grupo en la media (entre dimensión). Las primeras tienen como hipótesis nula la no cointegración $\left(H_{0}: \gamma_{i}=1 \forall i\right)$ y la alternativa $\left(H_{1}: \gamma_{i} \neq 1 \forall i\right)$. Las segundas tienen como hipótesis nula de no cointegración $\left(H_{0}: \gamma_{i}=1 \forall i\right)$ y la alternativa $\left(H_{1}: \gamma_{i} \neq 1 \forall i\right)$. La diferencia está en que en las segundas no necesariamente se cumple que $\gamma_{i}=\gamma$, de ahí que se suponga la heterogeneidad de las series cruzadas del panel de datos.

Los estadísticos del panel de cointegración (dentro de dimensión) son:

Estadístico- $v$ panel:

$$
T^{2} N^{\frac{3}{2}} Z_{\hat{v} N, T} \equiv T^{2} N^{\frac{3}{2}}\left(\sum_{i=1}^{N} \sum_{t=1}^{T} \frac{\hat{\varepsilon}_{i, t-1}^{2}}{\hat{\sigma}_{11 i}^{2}}\right)^{-1}
$$

Estadístico- $\rho$ panel:

$$
T \sqrt{N} Z_{\hat{\rho} N, T-1} \equiv T \sqrt{N}\left(\sum_{i=1}^{N} \sum_{t=1}^{T} \frac{\hat{\varepsilon}_{i, t-1}^{2}}{\hat{\sigma}_{11 i}^{2}}\right)^{-1} \sum_{i=1}^{N} \sum_{t=1}^{T} \frac{\hat{\varepsilon}_{i, t-1} \Delta \hat{\varepsilon}_{i, t}-\hat{\lambda}_{i}}{\hat{\sigma}_{11 i}^{2}}
$$

Estadístico- $t$ panel (no paramétrica PP):

$$
Z_{t N, T} \equiv\left(\tilde{\sigma}_{N, T}^{2} \sum_{i=1}^{N} \sum_{t=1}^{T} \frac{\hat{\varepsilon}_{i, t-1}^{2}}{\hat{\sigma}_{11 i}^{2}}\right)^{\frac{-1}{2}} \sum_{i=1}^{N} \sum_{t=1}^{T} \frac{\hat{\varepsilon}_{i, t-1} \Delta \hat{\varepsilon}_{i, t}-\hat{\lambda}_{i}}{\hat{\sigma}_{11 i}^{2}}
$$

Estadístico- $t$ panel (paramétrica ADF):

$$
\left(\tilde{S}_{N, T}^{2} \sum_{i=1}^{N} \sum_{t=1}^{T} \frac{\hat{\varepsilon}_{i, t-1}^{2}}{\hat{\sigma}_{11 i}^{2}}\right)^{\frac{-1}{2}} \sum_{i=1}^{N} \sum_{t=1}^{T} \frac{\hat{\varepsilon}_{i, t-1} \Delta \hat{\varepsilon}_{i, t}}{\hat{\sigma}_{11 i}^{2}}
$$


Los estadísticos del panel de cointegración del grupo en la media (entre dimensión) son:

Estadístico- $\rho$ grupo:

$$
T N^{\frac{-1}{2}} Z_{\hat{\rho} N, T-1} \equiv T N^{\frac{-1}{2}} \sum_{i=1}^{N}\left(\sum_{t=1}^{T} \hat{\varepsilon}_{i, t-1}^{2}\right)^{-1} \sum_{i=1}^{T} \hat{\varepsilon}_{i, t-1} \cdot \Delta \hat{\varepsilon}_{i, t}-\hat{\lambda}_{i}
$$

Estadístico- $t$ grupo (no paramétrica PP):

$$
N^{\frac{-1}{2}} Z_{t N, T-1} \equiv N^{\frac{-1}{2}} \sum_{i=1}^{N}\left(\hat{\sigma}_{i}^{2} \sum_{t=1}^{T} \hat{\varepsilon}_{i, t-1}^{2}\right)^{\frac{-1}{2}} \sum_{t=1}^{T} \hat{\varepsilon}_{i, t-1} \Delta \hat{\varepsilon}_{i, t}-\hat{\lambda}_{i}
$$

Estadístico- $t$ panel (paramétrica ADF):

$$
N^{\frac{-1}{2}} Z_{t N, T} \equiv N^{\frac{-1}{2}} \sum_{i=1}^{N}\left(\sum_{t=1}^{T} \tilde{s}_{i}^{2} \hat{\varepsilon}_{i, t-1}^{2}\right)^{\frac{-1}{2}} \sum_{t=1}^{T} \hat{\varepsilon}_{i, t-1} \Delta \hat{\varepsilon}_{i, t} .
$$

donde $T$ es el número de datos, $N$ es el número de secciones cruzadas, $\hat{\varepsilon}_{i, t}^{2}$ son los residuos de la regresión, $\hat{\sigma}_{11 i}^{2}$ es la varianza condicional de largo plazo de los

residuos

$$
\begin{gathered}
\hat{\varepsilon}_{i, t}^{2}+\frac{2}{T} \sum_{s=1}^{k_{i}}\left(1-\frac{s}{k_{i}+1}\right) \sum_{t=s+1}^{T} \hat{\varepsilon}_{i} \hat{\varepsilon}_{i, t-s} \\
\hat{\sigma}_{11 i}^{2}=\frac{1}{T} \sum_{t=1}^{T}
\end{gathered}
$$

(covarianza consistente con heteroce-

dasticidad y autocorrelación, HAC, por su siglas en inglés), $\hat{\lambda}_{i}$ es la diferencia entre las varianzas de largo y corto plazos (es el ajuste de la varianza), la cual se estima de dos formas, la primera de forma no paramétricas con $\hat{\varepsilon}_{i, t}=\hat{\gamma}_{i} \hat{\varepsilon}_{i, t-1}+\hat{u}_{i, t}$ para obtener la varianza de largo plazo $\left(\hat{\sigma}_{i}^{2}\right)$ de $\hat{u}_{i, t}$ y la varianza de corto plazo $\left(\hat{s}_{i}^{2}\right)$ de $\hat{u}_{i, t}$. De esta manera, $\hat{\lambda}_{i}=\frac{1}{2}\left(\hat{\sigma}_{i}^{2}-\hat{s}_{i}^{2}\right)$ ó $\hat{\lambda}_{i}=\frac{1}{T} \sum_{s=1}^{k_{i}}\left(1-\frac{s}{k_{i}+1}\right) \sum_{t=s+1}^{T} \hat{\varepsilon}_{i} \hat{\varepsilon}_{i, t-s}$. La

$$
\hat{\gamma}_{i, k} \hat{\varepsilon}_{i, t-k}+\hat{u}_{i, t}
$$

segunda forma es de tipo paramétrica y se estima

$$
\hat{\varepsilon}_{i, t}=\hat{\gamma}_{i} \hat{\varepsilon}_{i, t-1}+\sum_{k=1}^{K_{i}} \text { para obtener }
$$


la varianza de corto plazo $\left(\hat{s}_{i}^{2}\right)$ de $\hat{u}_{i, t}$. Por último, se define $\tilde{\sigma}_{N, T}^{2}=\frac{1}{2} \sum_{i=1}^{N} \frac{\hat{\sigma}_{i}^{2}}{\hat{\sigma}_{11 i}^{2}} \mathrm{y}$ $\tilde{S}_{N, T}^{2}=\frac{1}{2} \sum_{i=1}^{N} \hat{s}_{i}^{2}$

Por su parte, Maddala y Wu (1999) desarrollan una prueba de cointegración, retomando los resultados de Fisher (1932) y Johansen (1991). Fisher (1932) propone una prueba que puede combinar los resultados de pruebas individuales, mientras que Johansen (1991) propone la prueba de cointegración individual de vectores a través de:

$$
\text { Prueba de Traza o máximo eigenvalor }=-2 \sum_{i=1}^{N} \log \left(\pi_{i}\right) \rightarrow \chi_{2 N}^{2}
$$

donde $\pi_{i}$ es el $p$-value para cada prueba individual de cointegración de Johansen para la sección cruzada $i$.

\section{I.3. Minimos cuadrados ordinarios totalmente modificados}

La metodología extendida de cointegración para datos de panel se divide en dos partes. En la primera se estima el modelo de largo plazo de cada sección cruzada, o bien, el modelo agrupado para comparar los resultados, y así obtener los residuos. En la segunda parte se estima el modelo de corto plazo agrupado con base en el método de estimación de mínimos cuadrados ordinarios totalmente modificados propuesto por Phillips y Hansen (1990), el cual fue adaptado para datos de panel por Pedroni (1996, 2000), aunque también existen las propuestas de Phillips y Moon (1999) y Kao y Chiang (2000).

Pedroni $(1996,2000)$ propone una metodología para datos de panel para los tres diferentes tipos de modelos, es decir: modelo de datos agrupados, panel heterogéneo y grupo en la media; nosotros tomaremos el modelo de datos agrupados. De esta manera,

$$
\begin{aligned}
& y_{i t}=\alpha_{1}+\beta X_{i t}+\varepsilon_{1 t} \\
& X_{i t}=X_{i t-1}+\varepsilon_{2 t}
\end{aligned}
$$


donde $\xi_{i t}=\left(\varepsilon_{1 t}, \varepsilon_{2 t}\right)^{\prime}$ es el vector estacionario de los residuos con la matriz asintótica de covarianzas $\Omega_{1}$ de largo plazo (HAC) y estimada por algún método como el de Newey y West (1987) o Andrews (1991). También $\Omega_{1}$ se puede descomponer en $\Omega_{i}^{0}+\Gamma_{i}+\Gamma_{i}^{\prime}$, de manera que $\Omega_{i}^{0}$ es la covarianza actual y $\Gamma_{i}$ y $\Gamma_{i}^{\prime}$ representan la suma ponderada de las covarianzas. El estimador del parámetro $\beta$ está dado por:

$$
\begin{aligned}
& + \\
& +-\tilde{\Gamma}_{s t} \\
& \tilde{X}_{i t} \tilde{y}_{i t} \\
& \sum_{t=1}^{T} \\
& \hat{\beta}_{N T}=\left(\sum_{i=1}^{N} \sum_{t=1}^{T} \tilde{X}_{i t} \tilde{X}_{i t}^{\prime}\right)^{-1} \sum_{i=1}^{N}
\end{aligned}
$$

\section{Modelo de datos Panel CoIntegrado}

El horizonte de estudio abarca el periodo 1970-2015 y las unidades son los países que integran el TLCAN. Como se mencionó antes, las variables son PIB, CEE y VAM. ${ }^{6}$

${ }^{6}$ Algunos datos de la variable vam tuvieron que ser estimados suponiendo que el valor al momento . $t$.. está en función de un promedio anual de diez años anteriores dado el periodo 1970-2009. 
90 ECONOMÍA TeOrÍA y PrÁCTICA • Nueva Época, Número especial, vol. 4, agosto 2018

Gráfica 1. Variables de estudio en términos per cápita

\section{PIB per cápita}

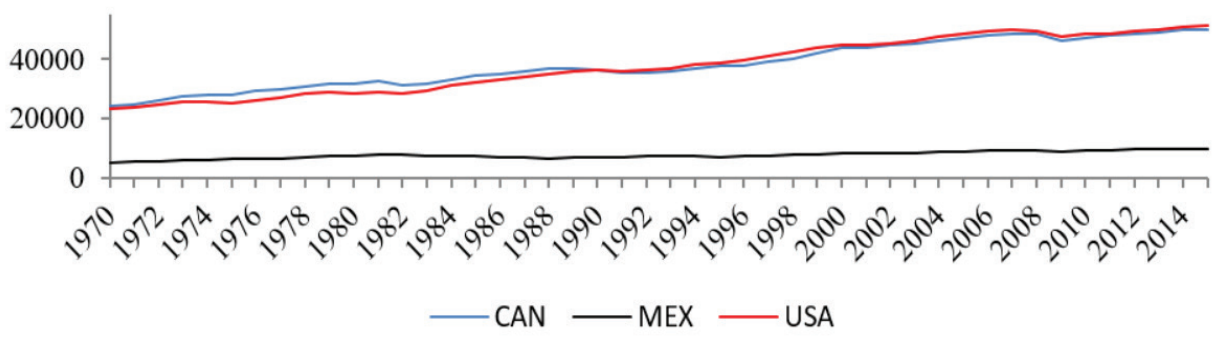

CEE per cápita

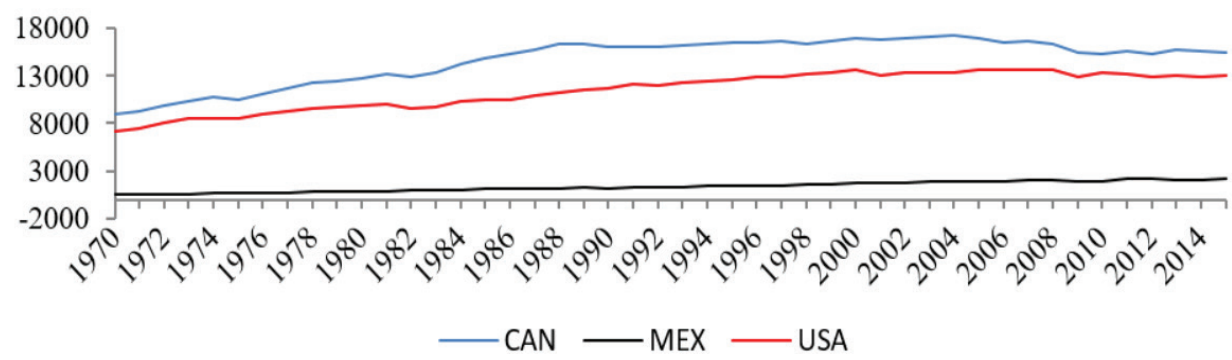

VAM per cápita

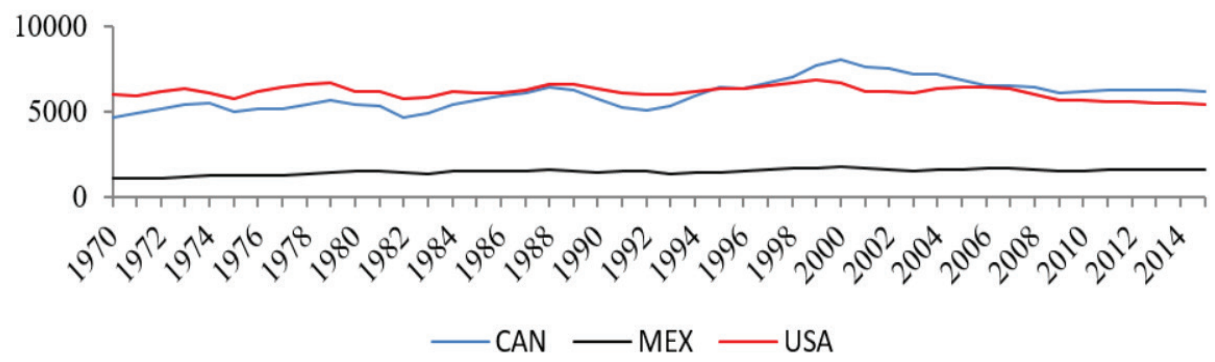

Fuente: elaboración propia con datos de la OCDE y el BM. 
Cuadro 1. Estadísticos básicos de las variables de estudio

\begin{tabular}{|c|c|c|c|c|c|c|c|c|c|c|c|c|c|}
\hline & & \multicolumn{2}{|c|}{ MEDIA } & \multicolumn{2}{|c|}{ DS } & \multicolumn{2}{|c|}{ SESGO } & \multicolumn{2}{|c|}{ CURTOSIS } & \multicolumn{2}{|c|}{$J-B$} & \multicolumn{2}{|c|}{ P } \\
\hline & & ANTES & DESPUÉS & ANTES & DESPUÉS & ANTES & DESPUÉS & ANTES & DESPUÉS & ANTES & DESPUÉS & ANTES & DESPUÉS \\
\hline \multirow{3}{*}{ PIB } & CAN & 31612 & 44587 & 3913 & 4537 & -0.3059 & -0.6495 & 2.0402 & 2.0150 & 1.2416 & 2.5467 & 0.5375 & 0.2799 \\
\hline & MEX & 6872 & 8674 & 764 & 849 & -0.3659 & \begin{tabular}{|c|}
-0.3583 \\
\end{tabular} & 2.4036 & 1.9760 & 0.8541 & 1.4970 & 0.6524 & 0.4731 \\
\hline & USA & 29823 & 45831 & 4317 & 4409 & 0.2179 & -0.6677 & 1.7559 & 2.1592 & 1.6652 & 2.3865 & 0.4349 & 0.3032 \\
\hline \multirow{3}{*}{ CEE } & CAN & 13070 & 16321 & 2466 & 619 & $\begin{array}{l}-0.0774 \\
\end{array}$ & -0.3756 & 1.6891 & 1.8715 & 1.6699 & 1.7612 & .4339 & 0.4145 \\
\hline & MEX & 888 & 1800 & 258 & 272 & -0.0770 & -0.5465 & 1.7092 & 2.0380 & 1.6193 & 2.0319 & 0.4450 & 0.3621 \\
\hline & USA & 9816 & 13146 & 1385 & 389 & -0.0344 & -0.3880 & 2.2053 & 2.6441 & 0.6098 & 0.6985 & 0.7372 & 0.7052 \\
\hline \multirow{3}{*}{ VAM } & CAN & 5424 & 667 & 472 & 657 & 0.4437 & 0.4554 & 2.6690 & 2.7883 & 0.8595 & 0.8381 & 0.6507 & 0.6577 \\
\hline & MEX & 1403 & 1601 & 162 & 95 & -0.6093 & -0.4118 & 2.0997 & 7881 & 2.2000 & 0.6931 & 0.3329 & 0.7071 \\
\hline & USA & 6211 & 6132 & 267 & 436 & 0.2178 & -0.1565 & 3013 & 1.8502 & 0.6498 & 1.3608 & 0.7226 & 0.5064 \\
\hline \multirow{3}{*}{ PIB } & CAN & 0.0172 & 0.0150 & 0.0246 & 0.0177 & -0.9404 & -1.2836 & 3.5374 & 6.2025 & 3.5073 & 15.4428 & 0.1731 & 0.0004 \\
\hline & MEX & 0.0144 & 0.0127 & 0375 & 0.0324 & $\begin{array}{l}-0.7892 \\
\end{array}$ & -1.4755 & 3.0497 & 4.9544 & 2.2862 & 11.4844 & 0.3188 & 0.0032 \\
\hline & USA & 2205 & 150 & 236 & 162 & $\begin{array}{l}-0.5452 \\
\end{array}$ & -1.5931 & 2.4298 & 9883 & 1.3879 & 17.4916 & 0.4996 & 0.0002 \\
\hline \multirow{3}{*}{ CEE } & CAN & 0.0266 & -0.0021 & .0280 & 0.0196 & -0.6550 & -0.9078 & 2.5865 & 4.0979 & 1.7297 & 4.1265 & 0.4211 & 0.1270 \\
\hline & MEX & 0.0445 & 0.0229 & 0.0271 & 0.0367 & -1.2103 & 0.0756 & 5.5854 & 2.6384 & 11.4986 & 0.1408 & 0.0032 & 0.9320 \\
\hline & USA & 0.0230 & 0.0027 & 0.0266 & 0.0222 & -0.4486 & -1.1836 & 3.3123 & 4.3098 & 0.8274 & 6.7094 & 0.6612 & 0.0349 \\
\hline \multirow{3}{*}{ VAM } & CAN & $36 \%$ & $0.71 \%$ & 630 & 0.0484 & -0.7919 & 0.5667 & 2.8802 & 2.5717 & 2.3128 & 1.3459 & 0.3146 & 0.5102 \\
\hline & MEX & $1.49 \%$ & $0.61 \%$ & 0.0395 & 0.0392 & -0.8529 & \begin{tabular}{|c|}
-0.1584 \\
\end{tabular} & 2.7376 & 3.1373 & 2.7303 & 0.1093 & 0.2553 & 0.9468 \\
\hline & USA & $0.01 \%$ & $-0.45 \%$ & 0.0429 & 0.0306 & -0.2078 & -0.9428 & 2.0477 & 3.6219 & 0.9897 & 3.6135 & 0.6097 & 0.1642 \\
\hline
\end{tabular}

Fuente: elaboración propia con datos de la OCDE y el BM, D-S desviación estándar, J-B Jarque-Bera, P probabilidad.

En la gráfica 1 se presentan las dinámicas de las series PIB, CEE y VAM en términos per cápita. Observe primero que en cuanto al PIB se distinguen para CAN y USA dos subperiodos. El primero abarca de 1970 hasta el principio de la década de los noventa, donde CAN tenía un PIB per cápita superior al de USA, después se invirtieron las tendencias, aunque permanecen muy cercanas y MEX es el país con menor PIB per cápita (representa aproximadamente el 21 por ciento del PIB anual per cápita de USA o CAN de dicho periodo). Por otro lado, las tasas de crecimiento antes y después del TLCAN son de 1.72 por ciento a 1.50 por ciento para CAN, 1.44 por ciento a 1.27 por ciento para MEX, y para USA son de 2.02 por ciento a 1.50 por ciento. Para el periodo 1970-1993 los ciudadanos de CAN, MEX y USA en promedio obtenían anualmente $31,612,6,872$ y 29,823 dólares, respectivamente, y tuvieron incrementos de 44,587, 8,674 y 45,821 dólares anuales en el periodo 1994-2015. El crecimiento esperado en el PIB no se cumplió, siendo usA el más afectado. En la segunda parte de la gráfica se observa el consumo de energía eléctrica: CAN es el país que más consume en términos per cápita, siguiéndole USA y al final MEX. La tasa de consumo de esta variable para MEX ha permanecido constante antes y después del TLCAN; en cambio, en CAN y USA ha disminuido a 2.66 por ciento y 2.30 por ciento de crecimiento anual promedio antes del tratado, y después de éste siguió disminuyendo hasta -0.21 por ciento y 0.27 por ciento, respectivamente. En cuanto al valor agregado de las manufacturas del total en términos per cápita, antes del TLCAN, crecía anualmente 0.36 por ciento, 1.49 por ciento y 0.01 por ciento para 
CAN, MEX y USA, respectivamente. Después del TLCAN, esta variable empeoró dado que se registró una caída en la tasa de crecimiento anual para MEX y una cifra negativa para USA de -0.45 por ciento. Obviamente esto se debe a factores externos, por ejemplo: no todas las partes que conforman un producto se crean dentro de los países del TLCAN, por lo tanto, existe traslado de valor agregado de un país a otro país; aunque para CAN se presentó un aumentó al 0.71 por ciento.

En el cuadro 1 se observa el comportamiento de las variables de estudio antes y después del TLCAN. Primero se distingue que CAN es el país que más se ha beneficiado con el TLCAN tomando en cuenta estas variables y en virtud de un aumento en su PIB per cápita, una disminución del CEE y un aumento del VAM en las tasas de crecimiento del periodo; el resto de los países han empeorado su situación. La mayor volatilidad en ambos periodos por parte de las variables de estudio la tiene MEX, en cambio, para CAN y USA ha disminuido con el tiempo. Los coeficientes de sesgo y curtosis son complementos porque, como se muestra en el cuadro 1, cuando el sesgo es mayor a la unidad (en términos absolutos y para este estudio en particular), la curtosis es muy superior a tres y el proceso se invierte cuando es inferior a la unidad. Por último, la prueba de normalidad Jarque-Bera muestra que

Cuadro 2. Pruebas de raíz unitaria de las series económicas

\begin{tabular}{|c|c|c|c|c|c|c|c|c|c|c|c|c|}
\hline \multirow{2}{*}{ Prueba } & $\mathrm{PIB}$ & P & CEE & $\mathrm{P}$ & VAM & $P$ & PIB & $\mathrm{P}$ & CEE & $\mathrm{P}$ & VAM & $P$ \\
\hline & \multicolumn{6}{|c|}{ Constante } & \multicolumn{6}{|c|}{ Constante y tendencia } \\
\hline \multicolumn{13}{|l|}{ Levin-Lin-Chu } \\
\hline Nivel & -2.37 & 0.01 & -6.90 & 0.00 & -2.15 & 0.02 & -0.95 & 0.17 & -1.18 & 0.12 & -1.85 & 0.03 \\
\hline Primera diferencia & -7.41 & 0.00 & -5.64 & 0.00 & -7.86 & 0.00 & -7.22 & 0.00 & -7.88 & 0.00 & -7.65 & 0.00 \\
\hline \multicolumn{13}{|l|}{ Hadri Z } \\
\hline Nivel & 7.96 & 0.00 & 7.62 & 0.00 & 4.96 & 0.00 & 1.42 & 0.08 & 6.28 & 0.00 & 3.07 & 0.00 \\
\hline Primera diferencia & 0.24 & 0.41 & 6.88 & 0.00 & 0.03 & 0.49 & 1.31 & 0.10 & 0.07 & 0.47 & -0.24 & 0.60 \\
\hline \multicolumn{13}{|l|}{ Im-Pesaran-Shin } \\
\hline Nivel & -0.31 & 0.38 & -4.64 & 0.00 & -2.02 & 0.02 & -0.90 & 0.18 & 1.64 & 0.95 & -1.52 & 0.06 \\
\hline Primera diferencia & \begin{tabular}{|l|}
-5.59 \\
\end{tabular} & 0.00 & -5.30 & 0.00 & -6.29 & 0.00 & -4.90 & 0.00 & \begin{tabular}{|l|}
-8.33 \\
\end{tabular} & 0.00 & -5.63 & 0.00 \\
\hline \multicolumn{13}{|l|}{ Fisher-ADF } \\
\hline Nivel & 5.08 & 0.53 & 32.92 & 0.00 & 13.54 & 0.04 & 8.33 & 0.22 & 1.04 & 0.98 & 10.64 & 0.10 \\
\hline Primera diferencia & \begin{tabular}{|l|}
41.20 \\
\end{tabular} & 0.00 & 38.86 & 0.00 & 47.78 & 0.00 & -0.97 & 0.17 & 61.40 & 0.00 & 38.63 & 0.00 \\
\hline \multicolumn{13}{|l|}{ Fisher-PP } \\
\hline Nivel & 6.68 & 0.35 & 42.69 & 0.00 & 12.91 & 0.04 & 4.03 & 0.67 & 0.93 & 0.99 & 4.19 & 0.65 \\
\hline Primera diferencia & 47.72 & 0.00 & 65.66 & 0.00 & 47.42 & 0.00 & 38.98 & 0.00 & 95.68 & 0.00 & 48.45 & 0.00 \\
\hline \multicolumn{13}{|l|}{ Choi-ADF } \\
\hline Nivel & -0.28 & 0.39 & -4.55 & 0.00 & -2.12 & 0.02 & 32.81 & 0.00 & 1.76 & 0.96 & -1.63 & 0.05 \\
\hline Primera diferencia & -5.32 & 0.00 & -5.07 & 0.00 & -5.85 & 0.00 & -4.54 & 0.00 & -6.85 & 0.00 & -5.06 & 0.00 \\
\hline \multicolumn{13}{|l|}{ Choi-PP } \\
\hline Nivel & \begin{tabular}{|l|}
-0.74 \\
\end{tabular} & 0.23 & -5.44 & 0.00 & -1.77 & 0.04 & 0.30 & 0.62 & 1.88 & 0.97 & 0.05 & 0.52 \\
\hline Primera diferencia & -5.85 & 0.00 & -7.09 & 0.00 & -5.82 & 0.00 & -5.13 & 0.00 & -8.89 & 0.00 & -5.76 & 0.00 \\
\hline
\end{tabular}

Fuente: elaboración propia con datos de la OCDE y el BM. P es la probabilidad crítica, si es mayor que 5.00 por ciento, se acepta la hipótesis nula de cada prueba. El tamaño del rezago fue elegido utilizando el criterio de información de Akaike. 
antes del tratado las variables se distribuían normalmente, pero después del TLCAN, el PIB per cápita es la variable que presenta problemas de normalidad, tomando en cuenta que la probabilidad debería ser mayor a 5.00 por ciento.

El cuadro 2 contiene los resultados de las pruebas de raíz unitaria y de estacionariedad mencionadas en la sección 2, las cuales se realizaron bajo dos supuestos; por un lado, se introdujo una constante y, por el otro, se buscó si las series seguían una tendencia. La prueba LLC rechaza la hipótesis nula para las tres variables de estudio. Por lo tanto, no tienen raíces unitarias y, en consecuencia, son estacionarias las variables en niveles y en diferencia cuando sólo se introduce la constante; en cambio, cuando se toma en cuenta la tendencia, sólo la primera diferencia rechaza la hipótesis nula. Con la prueba de Hadri (2000) de estacionariedad, las variables PIB y VAM son estacionarias en primeras diferencias, pero cuando se introduce una tendencia, todas las variables son estacionarias en primeras diferencias. Para la prueba IPS se tiene que todas las series rechazan la hipótesis nula cuando se toma la primera diferencia sin importar si se introdujo la tendencia o no. Las pruebas de Fisher-ADF y Fisher-PP rechazan la hipótesis nula con las series en primeras diferencias, a excepción del CEE cuando se toma sólo la constante. Por último, las pruebas de Choi-ADF y Choi-PP rechazan la hipótesis de raíz unitaria al 5 por ciento con la primera diferencia sin importar si tienen o no tendencia. En general, se observa que las variables son estacionarias cuando las pruebas se realizan con la primera diferencia.

Cuadro 3. Prueba de cointegración de Pedroni (1999 y 2004):

la variable endógena es PIB per cápita

\begin{tabular}{|c|c|c|c|c|c|}
\hline & Estadístico t & $P$ & Nivel & Estadístico t & $\mathrm{P}$ \\
\hline $\begin{array}{c}\text { Dentro } \\
\text { dimensión }\end{array}$ & & & & $\begin{array}{c}\text { Entre } \\
\text { dimensión }\end{array}$ & \\
\hline Estadístico & 1.48 & 0.07 & & & \\
\hline Estadístico & 0.90 & 0.82 & Grupo Estadístico & 1.52 & 0.94 \\
\hline Estadístico PP & 0.70 & 0.76 & Grupo Estadístico PP & 1.15 & 0.87 \\
\hline Estadístico ADF & -0.12 & 0.45 & Grupo Estadístico ADF & 0.46 & 0.68 \\
\hline \multicolumn{6}{|l|}{ Primera diferencia } \\
\hline Dentro dimensión & & & & Entre dimensión & \\
\hline Estadístico & -1.58 & 0.94 & & & \\
\hline Estadístico & -3.59 & 0.00 & Grupo Estadístico & -3.31 & 0.00 \\
\hline Estadístico PP & -4.44 & 0.00 & Grupo Estadístico PP & -5.10 & 0.00 \\
\hline Estadístico ADF & -2.78 & 0.00 & Grupo Estadístico ADF & -2.14 & 0.02 \\
\hline
\end{tabular}

Fuente: elaboración propia con datos de la OCDE y el BM. La hipótesis nula en las pruebas es que las variables no están cointegradas. Todas las variables están distribuidas normalmente $(0,1)$ bajo la hipótesis nula. 
94 ECONOMÍA TeORÍA y PrÁCTICA • Nueva Época, Número especial, vol. 4, agosto 2018

El cuadro 3 muestra las pruebas de cointegración de Pedroni (1999 y 2004). Si la prueba se realiza con las variables a nivel, sólo el estadístico " $v$ " rechaza la hipótesis nula al 10 por ciento. Por lo tanto, las variables están cointegradas en niveles, tomando en cuenta que ésta es la estadística de mayor parsimonia de las cuatro estadísticas dentro de dimensión. Por otro lado, todas las pruebas aplicadas a variables tomando la primera diferencia rechazan la hipótesis nula de no cointegración ya sea dentro o entre dimensiones. Por lo tanto, las variables están cointegradas en diferencias.

Cuadro 4. Resultados de la prueba de cointegración de Maddala y Wu (1999) para datos de panel, combinando la prueba de traza de Johansen (1988) y los resultados de Fisher (1932)

\begin{tabular}{|l|c|c|}
\hline & ESTADISTICO T NIVEL \\
\hline Ninguno & 30.24 & 0.00 \\
\hline Al menos 1 & 19.58 & 0.00 \\
\hline Al menos 2 & 9.06 & 0.17 \\
\hline & \multicolumn{2}{|c|}{ Primera diferencia } \\
\hline Ninguno & 61.56 & 0.00 \\
\hline Al menos 1 & 51.60 & 0.00 \\
\hline Al menos 2 & 49.31 & 0.00 \\
\hline
\end{tabular}

Fuente: elaboración propia con datos de la OCDE y el BM. La hipótesis nula en las pruebas es que las variables no están cointegradas.

Cuadro 5. Estimación por medio de mínimos cuadrados ordinarios (MCO) y mínimos cuadrados ordinarios totalmente modificados (мсотм)

\begin{tabular}{|c|c|c|c|c|c|c|c|}
\hline \multicolumn{5}{|c|}{ MCO } & \multicolumn{4}{c|}{ MCOTM } \\
\hline & C & CEE & VAM & & C & CEE & VAM \\
\hline CAN & -0.3754 & $0.6854^{*}$ & $0.4985^{*}$ & CAN & $0.1997^{*}$ & $0.5148^{* *}$ & $0.6227^{*}$ \\
\hline & {$[1.0029]$} & {$[0.1267]$} & {$[0.1691]$} & & {$[1.7956]$} & {$[0.2264]$} & {$[0.2879]$} \\
\hline MEX & $5.6946^{*}$ & $0.2923^{*}$ & 0.1604 & MEX & $6.4418^{*}$ & $0.3174^{*}$ & 0.0338 \\
\hline & {$[0.9023]$} & {$[0.0409]$} & {$[0.1558]$} & & {$[1.7510]$} & {$[0.0747]$} & {$[0.2972]$} \\
\hline USA & $4.4632^{*}$ & $1.3169^{*}$ & -0.7154 & USA & $6.1298^{*}$ & $1.3207^{*}$ & -0.9010 \\
\hline & {$[1.3546]$} & {$[0.0471]$} & {$[0.1472]$} & & {$[2.2088]$} & {$[0.0813]$} & {$[0.2366]$} \\
\hline Panel & $3.0107^{*}$ & $0.4369^{*}$ & $0.3873^{*}$ & Panel & $2.9209^{*}$ & $0.4291^{*}$ & $0.4066^{*}$ \\
\hline & {$[0.2872]$} & {$[0.0481]$} & {$[0.0823]$} & & {$[0.2786]$} & {$[0.0467]$} & {$[0.0798]$} \\
\hline
\end{tabular}

Fuente: elaboración propia con datos de la OCDE y el BM. La desviación estándar está entre corchetes, * 5 por ciento $y * * 10$ por ciento de significancia. 
En el cuadro 4 se muestran los resultados de la prueba de Maddala y Wu (1999), la cual combina la prueba de traza de Johansen (1988) con la de Fisher (1932). Esta prueba muestra que para las variables en nivel existe al menos un vector que está cointegrado. Por otro lado, la misma prueba aplicada a las variables tomando la primera diferencia lleva a que todas las series están cointegradas. Con los resultados de ambas pruebas se puede concluir que las series PIB, CEE y VAM tienen algún tipo de relación de corto y largo plazo.

A continuación, se estima la ecuación (2) por dos métodos: mínimos cuadrados ordinarios (MCO) y mínimos cuadrados ordinarios totalmente modificados (MCOTM). Además, se estimarán tanto individualmente como en el marco de datos de panel para indagar sobre la relación de largo plazo, para lo cual es indispensable considerar los residuos de las regresiones.

En el cuadro 5 se muestran los resultados de las estimaciones por medio de los métodos de estimación мсо у мсотм. Se observa primero la diferencia en los valores de los parámetros debido al ajuste que se realiza al introducir la correlación serial en la ecuación (17). Después, se observa que los parámetros tienen el signo esperado, a excepción de la variable vam para usa bajo los dos métodos de estimación. Posteriormente, se ve que la variable VAM no es significativa para MEX y USA al 5 por ciento, aunque para el modelo datos de panel sí lo es. Por último, la variable CEE es altamente significativa para explicar su relación con el PIB per cápita.

Para la segunda parte del método de cointegración Engle-Granger se proponen las siguientes ecuaciones donde se introduce el mecanismo de corrección de los errores:

$$
\begin{aligned}
& \triangle \text { LPIB }_{i t}=\alpha_{a 1}+\alpha_{a 2} \Delta L C E E_{i, t-1}+\alpha_{a 3} \Delta L V A M_{i, t-1}+\alpha_{a 4} \Delta L P I B_{i, t-1}+\alpha_{a 5} R_{i, t-1}+\varepsilon_{a i, t} \\
& \triangle L C E E_{i t}=\alpha_{b 1}+\alpha_{b 2} \Delta L C E E_{i, t-1}+\alpha_{b 3} \Delta L V A M_{i, t-1}+\alpha_{b 4} \Delta L P I B_{i, t-1}+\alpha_{b 5} R_{i, t-1}+\varepsilon_{b i, t} \\
& \Delta L V A M_{i t}=\alpha_{c 1}+\alpha_{c 2} \Delta L C E E_{i, t-1}+\alpha_{c 3} \Delta L V A M_{i, t-1}+\alpha_{c 4} \Delta L P I B_{i, t-1}+\alpha_{c 5} R_{i, t-1}+\varepsilon_{c i, t}
\end{aligned}
$$

donde $\Delta$ representa el operador de primera diferencia logarítmica de las variables de estudio, $R_{i, t}$ son los residuos obtenidos a partir de la ecuación (2) por el método мсотм о мсо, у su parámetro asociado representa la causalidad de largo plazo. Por otro lado, los parámetros asociados a las variables explicativas del modelo representan la causalidad de corto plazo. Se supone que los términos de error $\varepsilon_{a i, t}, \varepsilon_{b i, t}$ y $\varepsilon_{c i, t}$ no están correlacionados entre sí y tiene media cero. 
Cuadro 6. Prueba de causalidad para panel de datos

\begin{tabular}{|c|c|c|c|c|c|c|c|c|}
\hline $\begin{array}{c}\text { VARIABLE } \\
\text { DEPENDIENTE }\end{array}$ & \multicolumn{8}{|c|}{ VARIABLE INDEPENDIENTE } \\
\hline & \multicolumn{5}{|c|}{ CORTO PLAZO } & & \multicolumn{2}{|c|}{ LARGO PLAZO } \\
\hline \multicolumn{9}{|c|}{$\mathrm{MCO}$} \\
\hline & LPIB & p & LCEE & p & LVAM & P & R & P \\
\hline LPIB & & & 0.1004 & 0.7519 & 3.0479 & 0.0832 & 5.0468 & 0.0264 \\
\hline LCEE & 0.8943 & 0.3461 & & & 2.9424 & 0.0887 & 0.0473 & 0.8281 \\
\hline LVAM & 9.2956 & 0.0028 & 0.0271 & 0.8696 & & & 1.4902 & 0.2244 \\
\hline \multicolumn{9}{|c|}{ МСОTM } \\
\hline & LPIB & P & LCEE & P & LVAM & P & $R$ & $P$ \\
\hline LPIB & & & 0.0103 & 0.9191 & 2.7499 & 0.0997 & 3.3460 & 0.0697 \\
\hline LCEE & 1.0216 & 0.3140 & & & 3.2133 & 0.0754 & 4.4925 & 0.0360 \\
\hline LVAM & 8.5726 & 0.0040 & 0.0353 & 0.8512 & & & 2.3882 & 0.1247 \\
\hline
\end{tabular}

Fuente: elaboración propia con datos de la OCDE y el BM. P es la probabilidad. La hipótesis nula, la variable independiente no causa en el sentido Granger a las variables dependientes.

En el cuadro 6 se muestran los resultados de la prueba de causalidad de Granger para el modelo de datos de panel usando мсо у мсотм, lo cual permite obtener relaciones causales de corto y largo plazo de las variables. Adicionalmente, la prueba bajo la hipótesis nula supone que el modelo es agrupado y no es un modelo de efectos fijos, dado que supone que los parámetros son iguales para todas las secciones cruzadas. Prácticamente las dos metodologías de estimación ofrecen resultados similares, a excepción de la relación causal de largo plazo de la variable CEE, donde мCо acepta la hipótesis nula у мсотм la rechaza. En el corto plazo se observa que el crecimiento económico es explicado por el vAM, dado que rechaza la hipótesis nula de no relación causal en el sentido Granger al 10 por ciento. De forma similar se observa en el largo plazo dado que el modelo de la ecuación (2) sí explica los movimientos de PIB. Además, se observa una relación bidireccional entre el vAM y PIB. Por otro lado, la variable CEE mantiene una relación unidireccional con VAM, dado que esta última explica a la primera variable. El CEE no tiene el mismo desempeño porque se acepta la hipótesis nula. Por lo tanto, los resultados coinciden con las pruebas de cointegración de los cuadros 3 y 4 , los cuales mencionan que al menos un vector está cointegrado; en este caso, la variable vaM. Por último, los resultados obtenidos de la prueba de causalidad muestran que la variable CEE no tiene relación causal con el crecimiento económico o con la tasa de crecimiento del VAM, aunque 
sí tiene efectos en el largo plazo. Para finalizar el estudio a continuación se muestra la gráfica 2 con los residuos recursivos para estudiar la estabilidad de los parámetros de los modelos de corto plazo de los métodos de estimación мсо у мсотм.

Gráfica 2. Residuos recursivos de los modelos мсотм у мсо

\section{CAN}

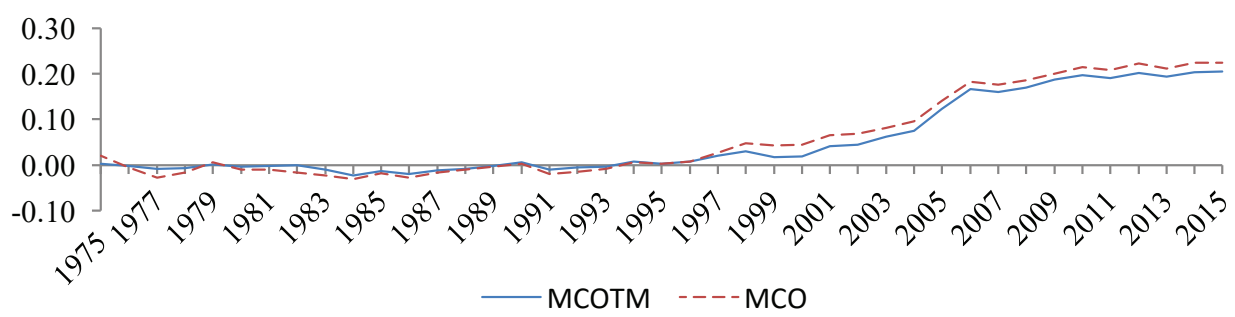

MEX

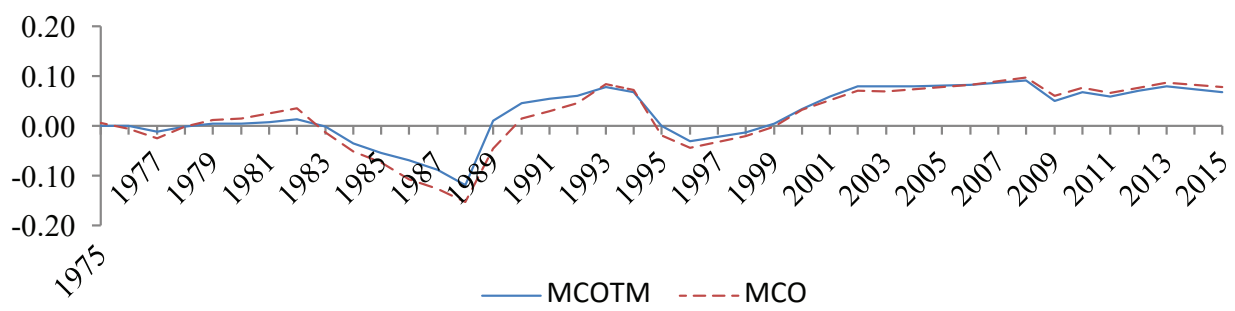

USA

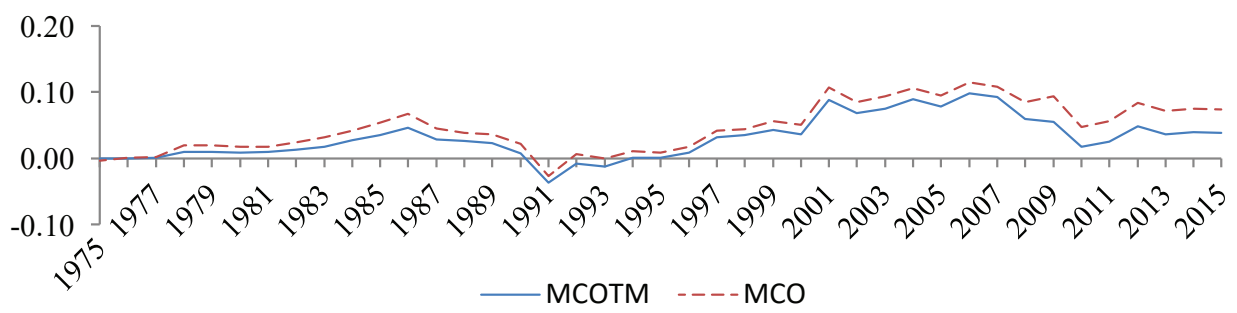

Fuente: elaboración propia con datos de la OCDE y el BM. 
En la gráfica 2 se puede apreciar la similitud de movimientos de los residuos recursivos obtenidos por ambas metodologías de estimación. Por otro lado, CAN, MEX y USA tienen un cambio estructural después de la firma del tratado de libre comercio en 1993, aunque México en sentido contrario, ya que se están sobreestimando los parámetros. Los otros cambios estructurales coinciden con varias crisis económicas, por ejemplo, en MEX 1982 y USA 2008. En general, CAN tiene los parámetros más estables durante el periodo de estudio, sólo viéndose afectados después del tratado.

Cuadro 7. Prueba de F para cambio estructural

\begin{tabular}{|l|l|l|l|}
\hline & CAN & MEX & USA \\
\hline 1988 & 15.2788 & 22.1300 & 9.7920 \\
\hline 1993 & 23.5032 & 12.2682 & 31.1357 \\
\hline 2000 & 366.0224 & 11.4611 & 38.4020 \\
\hline
\end{tabular}

Fuente: elaboración propia con datos de la OCDE y el BM.

En los resultados de la prueba $F$ se ponderan los residuos del modelo sin restricciones y con restricciones dados los puntos de cambio estructural que se obtuvieron en la prueba que fortalece lo observado en la gráfica 2. Es decir, sí existe un cambio estructural después de la firma del tratado de libre comercio entre los países firmantes.

\section{Conclusiones}

En esta investigación se estudió el comportamiento de la variación del PIB per cápita de los países que integran el TLCAN. Las variables explicativas fueron CEE y VAM en términos per cápita. La metodología utilizada fue la de cointegración, propuesta por Engle y Granger (1987), extendida para datos de panel. Las pruebas de raíz unitaria y de estacionariedad para datos de panel de la sección 2 muestran que las variables de estudio son $I(1)$. Asimismo, se aplicaron las pruebas de cointegración y los resultados mostraron que existe al menos un vector cointegrado (cuadros 3 y 4). Posteriormente, se realizó la estimación de la ecuación (2) por dos métodos, мСо у мСотм, los cuales mostraron que, aunque existía diferencia en los valores de los parámetros, coincidían con la significancia de éstos. Los resultados del modelo propuesto en la ecuación (18) muestra que existe, en el largo plazo, una relación bidireccional entre las variables PIB per cápita y vam per cápita, es decir, la I+D que tienen las manufacturas influyen positivamente en el crecimiento económico y viceversa. En cambio, el CEE per cápita tiene una relación neutral en el corto plazo con VAM. Sin embargo, estas dos variables en el largo plazo influyen positivamente 
sobre la variable a explicar. Por último, se examinó la presencia de algún cambio estructural en el modelo de largo plazo, para lo cual se estimaron los residuos recursivos, encontrando que sí existió un cambio estructural después de la firma del tratado de libre comercio, lo cual se confirmó con la prueba $F$ del cuadro 7. Para futuras investigaciones se introducirán variables que capturen mejor ese rompimiento estructural, ya que las pruebas mostraron que existía relación de corto y largo plazo con el uso del modelo agrupado en el que las economías tienen comportamiento similar.

\section{REFERENCIAS BIBLIOGRÁFICAS}

Aali-Bujari, Alí; Venegas-Martínez, Francisco y Palafox-Roca, Alfredo Omar (2017), "Impact of Energy Consumption on Economic Growth in Major OECD Economies (1977-2014): A Panel Data Approach", International Journal of Energy Economics and Policy, 7 (2), pp. 18-25.

Aghion, Philippe; Blundell, Richard; Griffith, Rachel; Howitt, Peter y Prantl, Susanne (2004), "Entry and Productivity Growth: Evidence from Microlevel Panel Data", Journal of the European Economic Association, 2 (2-3), pp. 265-276.

Andrews, Donald W. K. (1991), "Heteroskedasticity and Autocorrelation Consistent Covariance Matrix Estimation", Econometrica, 58 (3), pp. 817-858.

Akinlo, Anthony E. (2009), "Electricity Consumption and Economic Growth in Nigeria: Evidence from Cointegration and Co-Feature Analysis", Journal of Policy Modeling, 31 (5), pp. 681-693.

Apergis, Nicholas y Payne, James E. (2010), "Energy Consumption and Growth in South America: Evidence from a Panel Error Correction Model", Energy Economics, 32 (6), pp. 1421-1426.

Bhattacharya, Milta; Reddy Paramati, Sudharshan; Ozturk, Ilhan and Bhattacharya, Sankar (2016), "The Effect of Renewable Energy Consumption on Economic Growth: Evidence from Top 38 Countries", Applied Energy, 162 (C), pp. 733-741.

Choi, In (2001), "Unit Root Tests for Panel Data", Journal of International Money and Finance, 20 (2), pp. 249-272.

Coers, Robin y Sanders, Mark (2013), "The Energy-GDP Nexus; Addressing an Old Question with New Methods", Energy Economics, 36 (C), pp. 708-715.

Destek, Mehmet A. (2016), "Natural Gas Consumption and Economic Growth: Panel Evidence from oECD Countries”, Energy, 114 (C), pp. 1007-1015. 
Dickey, David A. y Fuller, Wayne A. (1979), "Distribution of The Estimators for Autoregressive Time Series with a Unit Root", Journal of the American Statistical Association, 74 (366a), pp. 427-431.

Engle, Robert F. y Granger, Clive W. J. (1987), "Co-Integration and Error Correction: Representation, Estimation, and Testing”, Econometrica, 55 (2), pp. 251-276.

Fatai, Osaiasi Koli; Oxley, Les y Gordon Scrimgeour, Francis (2004), "Modelling the Causal Relationship between Energy Consumption and GDP in New Zealand, Australia, India, Indonesia, the Philippines and Thailand", Mathematics and Computers in Simulation, 64 (3), pp. 431-445.

Fisher, Ronald Aylmer (1932), Statistical Methods for Research Workers. 5th ed., rev. and enl., Edinburgh, Oliver and Boyd.

Ghosh, Sajal (2010), "Examining Carbon Emissions Economic Growth Nexus for India: A Multivariate Cointegration Approach", Energy Policy, 38 (6), pp. 3008-3014.

Hadri, Kaddour (2000), "Testing for Stationarity in Heterogeneous Panel Data", The Econometrics Journal, 3 (2), pp. 148-161.

Hamilton, James D. (1994), Time Series Analysis, vol. 2, Princeton, Princeton University Press.

Im, Kyung So; Pesaran, M. Hashem y Shin, Yongcheol (2003), "Testing for Unit Roots in Heterogeneous Panels", Journal of Econometrics, 115 (1), pp. 53-74.

Johansen, Søren (1988), "Statistical Analysis of Cointegration Vectors", Journal of Economic Dynamics and Control, 12 (2-3), pp. 231-254.

(1991), "Estimation and Hypothesis Testing of Cointegration Vectors in Gaussian Vector Autoregressive Models", Econometrica, 59 (6), pp. 1551-1580.

Kao, Chihwa y Chiang, Min-Hsien (2000), "Nonstationary Panels, Panel Cointegration and Dynamic Panels", Advances in Econometrics, 6 (5), pp. 179-222.

Kasman, Adnan y Duman, Yavuz Selman (2015), "CO2 Emissions, Economic Growth, Energy Consumption, Trade and Urbanization in New EU Member and Candidate Countries: A Panel Data Analysis", Economic Modelling, 44 (C), pp. 97-103.

Kraft, John y Kraft, Arthur (1978), "On the Relationship between Energy and GNP”, The Journal of Energy and Development, 23 (8), pp. 401-403. 
Kwiatkowski, Denis; Phillips, Peter C. B.; Schmidt, Peter y Shin, Yongcheol (1992), "Testing the Null Hypothesis of Stationarity against the Alternative of a Unit Root: How Sure Are We that Economic Time Series Have a Unit Root?, Journal of Econometrics, 54 (1-3), pp. 159-178.

Kuznets, Simon (1966), Modern Economic Growth, Yale University Press, New Haven.

Levin, Andrew; Lin, Chien-Fu y James Chu, Chia-Shang (2002), "Unit Root Tests in Panel Data: Asymptotic and Finite-Sample Properties", Journal of Econometrics, 108 (1), pp. 1-24.

Maddala, Gangadharrao Soundalyarao y Wu, Shaowen (1999), "A Comparative Study of Unit Root Tests with Panel Data and a New Simple Test", Oxford Bulletin of Economics and Statistics, 61 (S1), pp. 631-652.

Narayan, Paresh Kumar y Prasad, Arti (2008), "Electricity Consumption-Real GDP Causality Nexus: Evidence from a Bootstrapped Causality Test for 30 OECD Countries", Energy Policy, 36 (2), pp. 910-918.

Newey, Whitney K. y West, Kenneth D. (1987), "Hypothesis Testing with Efficient Method of Moments Estimation", International Economic Review, 28 (3), pp. 777-787.

Ozcan, Burku (2013), "The Nexus between Carbon Emissions, Energy Consumption and Economic Growth in Middle East Countries: A Panel Data Analysis", Energy Policy, 62 (C), pp. 1138-1147.

Ozturk, Ilhan (2010), "A Literature Survey on Energy-Growth Nexus", Energy policy, 38 (1), pp. 340-349.

Parsons, Stanley Alfred James (1968), The Framework of Technical Innovation, Macmillan, London.

Pedroni, Peter (1999), "Critical Values for Cointegration Tests in Heterogeneous Panels with Multiple Regressors", Oxford Bulletin of Economics and Statistics, 61 (s1), pp. 653-670.

(1996), "Fully Modified OLS for Heterogeneous Cointegrated Panels and the Case of Purchasing Power Parity", Manuscript, Department of Economics, Indiana University. (2000), "OLS completamente modificado para paneles cointegrados heterogéneos", Advances in Econometrics, 15, pp. 93-130.

(2001), "Purchasing Power Parity Tests in Cointegrated Panels", Review of Economics and Statistics, 83 (4), pp. 727-731. 
(2004). "Panel Cointegration: Asymptotic and Finite Sample Properties of Pooled Time Series Tests with an Application to the PPP Hypothesis", Econometric Theory, 20 (03), pp. 597-625.

Phillips, Peter C. B. y Hansen, Bruce E. (1990), "Statistical Inference in Instrumental Variables Regression with I (1) Processes", The Review of Economic Studies, 57 (1), pp. 99-125.

Phillips, Peter C. B. y Moon, Hyungsik R. (1999), "Linear Regression Limit Theory for Nonstationary Panel Data”, Econometrica, 67 (5), pp. 1057 1111.

Phillips, Peter C. B., y Perron, Pierre (1988), "Testing for a Unit Root in Time Series Regression”, Biometrika, 75 (2), pp. 335-346.

Rezitis, Anthony N. R. y Ahammad, Shaikh Mostak (2016), "Energy Consumption and Economic Growth in South and Southeast Asian Countries: Evidence from a Dynamic Panel Data Approach”, International Energy Journal, 15 (3), pp. 103-116.

Salazar-Núñez, Héctor Francisco y Venegas-Martínez, Francisco (2018), “Impacto del uso de energía y la formación bruta de capital en el crecimiento económico: un análisis de datos panel en 73 países agrupados por nivel de ingreso", El Trimestre Económico, 85 (2), núm. 338, pp. 341-364.

Schmookler, Jacob (1966), Invention and Economic Growth, Harvard University Press, Cambridge, Mass.

Schumpeter, Joseph Alois (1911), The Theory of Economic Development: An Inquiry into Profits, Capital, Credit, Interest, and the Business Cycle, translated from the German, New Brunswick (USA) and London (UK).

Shahbaz, Muhammad; Tang Chor Foon y Shabbir, Muhammad Shahbaz (2011), "Electricity Consumption and Economic Growth Nexus in Portugal Using Cointegration and Causality Approaches", Energy Policy, 39 (6), pp. 3529-3536.

Tang, Chor Foon y Tan, Eu Chye (2013), "Exploring the Nexus of Electricity Consumption, Economic Growth, Energy Prices and Technology Innovation in Malaysia", Applied Energy, 104 (c), pp. 297-305.

Yoo, Seung-Hoon (2005), "Electricity Consumption and Economic Growth: Evidence from Korea", Energy Policy, 33 (12), pp. 1627-1632.

Yuan, Jia-Hai; Kang, Jian-Gang; Zhao, Chang-Hong y Hu, Zhao-Guang (2008), "Energy Consumption and Economic Growth: Evidence from China at both Aggregated and Disaggregated Levels", Energy Economics, 30 (6), pp. 3077-3094. 